\title{
Médiévales
}

Langues, Textes, Histoire

77 | automne 2019

Mathématiques. Savoirs et enseignements (OrientOccident)

\section{Les cautelae : un corpus de problèmes mathématiques entre collection, série et culture mathématique}

Cautelae: A Corpus of Mathematical Prob-lems, between Collection, Series and Mathematical Culture

Stéphane Lamassé

\section{(2) OpenEdition}

Journals

Édition électronique

URL : https://journals.openedition.org/medievales/10356

DOI : 10.4000/medievales. 10356

ISSN : 1777-5892

Éditeur

Presses universitaires de Vincennes

Édition imprimée

Date de publication : 5 décembre 2019

Pagination : 75-96

ISBN : 978-2-37924-061-4

ISSN : 0751-2708

Référence électronique

Stéphane Lamassé, "Les cautelae : un corpus de problèmes mathématiques entre collection, série et culture mathématique », Médiévales [En ligne], 77 | automne 2019, mis en ligne le 01 janvier 2022 consulté le 22 avril 2022. URL : http://journals.openedition.org/medievales/10356 ; DOI : https:// doi.org/10.4000/medievales.10356 


\section{Les cautelae : un corpus de problèmes mathématiques entre collection, série et culture mathématique}

L'activité mathématique du Moyen Âge occidental a laissé une assez grande variété de textes, encore bien souvent évalués aujourd'hui pour leur caractère savant ou non. Un certain nombre d'entre eux présentent des problèmes décrits comme récréatifs ${ }^{1}$, soit jugés comme " non applicatifs » ou simplement amusants. Une tradition de compilation de ces problèmes apparaît dès le haut Moyen Âge occidenta $1^{2}$. Cette étude entend contribuer à l'analyse d'un ensemble de documents que l'on peut dater du début du $\mathrm{XIII}^{\mathrm{e}}$ siècle $^{3}$. Il s'agit de listes de problèmes qui ne sont pas mis dans un contexte mathématique particulier : ils ne disposent ni d'introduction ni de règles expliquant les procédures. La composition de ces ensembles

1. L'idée de récréation et le champ lexical qui lui est associé comme celui de l' «énigme», renvoient à des points qui semblent importants pour les mathématiciens parce qu'ils sont liés à la pratique d'un savoir, au dévoilement et au défi qu'il n'est pas le lieu de développer ici, mais qu'il faut garder à l'esprit.

2. Ces textes ont assez vite été placés sous des noms comme ceux de Bède le Vénérable ou d'Alcuin. On trouvera dans F. CAJORI, A History of Elementary Mathematics with Hints on Methods of Teaching, New York, 1917, p. 113 une attestation de l'intérêt pour les Propositiones ad acuendos juvenes, à propos duquel il est dit que : "The collector of these problems evidently aimed to entertain and please his readers. » Plus largement, l'intérêt pour les problèmes et leur circulation appartient à la relation que l'on peut tisser entre les mathématiques et une dimension ludique de l'apprentissage des savoirs. On trouve dans un ouvrage récent et présentant d'une façon générale les grands textes de l'histoire des mathématiques médiévales un passage succinct sur les problèmes attribués à Alcuin et de leur influence dans l'Occident médiéval. Il s'agit, ici, davantage d'études de traditions textuelles, nous reviendrons sur cet aspect. Voir V. J. Katz, M. Folkerts, B. B. Hughes, R. Wagner, J. L. BergGRen, Sourcebook in the Mathematics of Medieval Europe and North Africa, New Jersey, 2018, p. 57.

3. C'est une réflexion qui a pu profiter d'un séminaire organisé dans le cadre du Labex Hastec et qui a donné lieu à une publication : A. BERNARD, « Les séries de problèmes, un genre au carrefour des cultures : une première synthèse », SHS Web of Conferences, 22 (2015), [en ligne] DOI: $10.1051 /$ shsconf/20152200001. 
de problèmes repose sur un principe de succession, sans que cela soit systématique. Lorsqu'ils possèdent un intitulé, celui-ci renvoie plutôt à des jeux d'esprits, comme subtilitates, cautelae, ludii, enigmata ou encore astucie $^{4}$. L'association occasionnelle de ces dénominations reflète, d'ailleurs, une certaine cohérence sémantique correspondant à ce type de pratique ${ }^{5}$. La présence occasionnelle du complément du nom algorismi atteste parfois d'un lien avec une activité mathématique pouvant appartenir à la culture universitaire $^{6}$. La compréhension de ces collections, entendues comme des regroupements de problèmes susceptibles de partager des points communs, reste encore aujourd'hui difficile à comprendre et demeure peu interrogée.

Je chercherai à montrer ici que cet ensemble documentaire constitue un groupe cohérent qu'il est intéressant de considérer pour lui-même. À travers lui, j'aborderai le développement, sur une période assez longue, d'un aspect de la culture mathématique occidentale caractérisé par des références à ces problèmes et par leur utilisation. Cela nécessite de revenir tout d'abord sur les différentes approches historiographiques, parce qu'elles façonnent en partie les appréciations actuelles de ces recueils. Cet examen nous invite à reconsidérer l'ensemble de ces textes qui forment un corpus dont le nombre d'exemplaires qui nous sont parvenus atteste d'une diffusion notable. On essaiera d'observer s'il est possible de dégager de ces documents une structure susceptible de refléter un modèle auquel ils pouvaient chercher à ressembler et dont le sens et les finalités ont évolué. Cela nous permettra d'envisager ce que la lecture de ces textes peut nous apporter sur les usages dont ils ont pu faire l'objet, en montrant comment ces recueils ont été constamment réappropriés. La dernière partie de cette étude se concentrera donc sur les relations intertextuelles que l'on peut établir entre ces recueils et d'autres textes, en particulier des traités, afin d'observer, serait-ce rapidement, comment ces problèmes ont pu être utilisés comme support à d'autres types d'activité mathématique.

\section{Des récréations aux recueils : la part de l'historiographie}

\section{Désarticuler les recueils pour mettre les problèmes en catalogue}

On connaît pour le Moyen Âge occidental des ouvrages ou des opuscules dont la structure repose sur le regroupement de problèmes. Une partie d'entre eux a déjà fait l'objet d'éditions ${ }^{7}$. En 1895, Maximilian

4. Ce que l'on pourrait traduire par subtilités, ruses, jeux, énigmes et astuces.

5. On peut prendre l'exemple du ms. London, British Library, Egerton 2622, f. 168v : « cautelas que alias enigmatur dicatur ». Il y a là une forme de synecdoque.

6. Ceci avait déjà été observé dans G. BEAujouAn, «L'enseignement de l'arithmétique élémentaire à l'Université de Paris aux XIII et XIV siècles ", dans Homenaje a MillásVallicrosa, Barcelone, t. I, 1954, p. 93-124.

7. Nous n'en ferons pas l'inventaire ici. Nous avons déjà signalé les Propositiones ad acuendos juvenes (éditées par M. FolKerTs dans Alcuin, Die älteste mathematische 
Curtze publie un texte du XIV ${ }^{\mathrm{e}}$ siècle, conservé à Munich, collectionnant 34 problèmes d'arithmétiques ${ }^{8}$. L'ensemble des énoncés et des méthodes de résolution peuvent être de nature assez variée ${ }^{9}$. Pour M. Curtze, le document relève sans ambiguïté d'une pratique ludique. Il ne souligne pas qu'il s'inscrit également dans un ensemble quantitativement plus important, celui des cautelae, qui abrite des problèmes dont certains ont eu une influence considérable. Repris et réutilisés sur une longue durée ${ }^{10}$, ces problèmes sont devenus presque patrimoniaux, au sens où ils constituent des références largement utilisées et partagées, parfois même par-delà le domaine des mathématiques. En mettant à disposition les textes constitutifs d'une discipline encore naissante sur le plan institutionnel, l'édition de Curtze répond à une logique historiographique. Elle s'inscrit en outre dans le cadre de recherches sur l'apprentissage et la circulation des savoirs mathématiques au Moyen Âge ${ }^{11}$. C'est avec cette approche généalogique que M. Curtze, Kurt Vogel et David E. Smith utilisent les problèmes dans

Aufgabensammlung in lateinischer Sprache : die Alkuin zugeschriebenen Propositiones ad acuendos iuvenes. Überlieferung, Inhalt, kritische Edition, Vienne, 1978). On peut ajouter le De arithmeticis propositionibus du pseudo-Bède (édité lui aussi par M. FoLKERTS dans Pseudo-Beda, De arithmeticis propositionibus : eine mathematische Schrift aus der Karolingerzeit, Wiesbaden, 1972). Pour le $\mathrm{xv}^{\mathrm{e}}$ siècle, il y a par exemple le Die pratica des algorismus ratisbonensis composé de 354 problèmes dont certains peuvent avoir un caractère applicatif et non ludique (édité par K. Vogel, Die Practica des Algorismus Ratisbonensis : ein Rechenbuch des Benediktinerklosters St. Emmeram aus der Mitte des 15. Jahrhunderts nach den Handschriften der Münchner Staatsbibliothek und der Stiftsbibliothek St. Florian, Munich, 1954). On peut aussi mentionner l'édition d'un recueil du XIII e siècle par J. SESIANO, "Un recueil du XIII" siècle de problèmes mathématiques », SCIAMVS, 1 (2000), p. 71-132, que nous utiliserons à titre de comparaison dans la suite de cet article.

8. Il s'agit du ms. München, lat. 14684 (Clm. 14684), [en ligne] http://daten.digitalesammlungen.de/bsb00086348/image_61 [consulté le 17 juillet 2019]. L'incipit (« Incipit subtilitates enigmatum ») fait aussi bien référence à la curiosité qu'au récréatif : M. CuRTZE, " Arithmetische Scherzaufgaben aus dem 14. Jahrhundert », Bibliotheca Mathematica, NF 9 (1895), p. 77-88.

9. On trouve dans cet ensemble des énoncés comme celui du chou, de la chèvre et du loup devant traverser une rivière ; un autre concernant l'achat de 20 oiseaux de trois sortes, pour 20 deniers ; ou bien encore l'estimation de la quantité de deniers qu'un associé a dans sa bourse, et d'autres devinettes comme celles de l'anneau, ainsi que des problèmes de transvasements. Pour plus de détails, voir J. Sesiano, Récréations mathématiques au Moyen Âge, Lausanne, 2014, où pratiquement tous ces problèmes sont analysés.

10. L'intérêt des mathématiciens pour ces collections est ancien. Pour s'en faire une idée on peut consulter K. Chemla, « Explorations in the History of Mathematical Recreations : An Introduction », Historia Mathematica, 41/1 (2014), p. 367-376 ; ou É. BARBIN, C. GolDSTEIN, M. Moyon, S. R. Schwer, S. Vinatier éd., Les Travaux combinatoires en France (1870-1914) et leur actualité : un hommage à Henri Delannoy, Limoges, 2017.

11. La dimension pédagogique des problèmes est un aspect assez important pour comprendre une part de l'intérêt que ces problèmes ont suscité. C'est ainsi que F. CAJORI présente les Propositiones ad acuendos juvenes, tout en essayant de retracer les influences de Héron, des Indiens et de l'Anthologie grecque (A History of Elementary Mathematics..., p. 113). 
leurs publications ${ }^{12}$. Menso Folkerts revient sur cet héritage en 1971 et propose une comparaison entre 33 recueils similaires à ceux publiés par Curtze. Sa démarche quantitative lui permet de regrouper les variantes en 10 types, qu'il identifie à partir d'une comparaison des mathématiques mises en œuvre et des traditions dans lesquelles elles s'insèrent. Pour chacun d'eux, M. Folkerts indique également la place qu'il occupe dans le recueil. Le classement auquel il aboutit est le suivant (Tableau 1):

1. Deviner les chiffres.

2. Problèmes de convivialité, il s'agit de problèmes indéterminés.

3. Problèmes linéaires du premier degré.

- l'âge du fils

- la lance dans l'eau

4. Prendre et donner.

5. L'épervier.

6. Le gardien dans le jardin.

- le pauvre et les offrandes

- le gardien dans le verger

- le testament

7. Suites arithmétiques et géométriques.

8. Problème de transport.

9. La distribution de vin.

10. Problèmes plaisants.

- Un vendeur achète 1000 oiseaux

— Trois frères doivent vendre des poires.

Tableau 1 : La typologie des cautelae adoptée par Menso Folkerts.

Pour l'historien allemand, l'étude de ces problèmes est susceptible de livrer un éclairage sur les changements intervenus dans les approches mathématiques entre les écoles des monastères et celles des villes, entre les cathédrales et les universités. Cette idée fait écho à celle de Guy Beaujouan ${ }^{13}$. Pour autant, M. Folkerts n'utilise ni l'ordonnancement des problèmes, ni les séries que ces recueils constituent pour en étudier les variantes. Il délaisse également la dimension diachronique pour se concentrer sur les « problèmes

12. D. E. Sмітн, « On the Origin of Certain Typical Problems », The American Mathematical Monthly, 24 (1917), p. 64-71.

13. G. Beaujouan, « L'enseignement de l'arithmétique élémentaire à 1'Université de Paris... ». C'est sans doute une des raisons pour lesquelles M. Folkerts a proposé par la suite une édition du De arithmeticis propositionibus du pseudo-Bède (De arithmeticis propositionibus : eine mathematische Schrift...) et des Propositiones ad acuendos juvenes attribuées à Alcuin (Die älteste mathematische Aufgabensammlung in lateinischer Sprache...). 
les plus importants ${ }^{14} \gg$. Cette démarche se traduit dans le second $\mathrm{xx}^{\mathrm{e}}$ siècle par la construction d'un catalogue de problèmes ${ }^{15}$. Il s'agit de concilier la synthèse et l'outil de recherche. Les problèmes sont souvent communs à un grand nombre de cultures intellectuelles et cela justifie que l'on souhaite en dresser un état, en faire d'abord un catalogue susceptible de favoriser des comparaisons. Il faut organiser une exploration intertextuelle dont le pivot est le problème. Et pour cela, il est nécessaire de les ordonner selon des critères, c'est-à-dire de constituer une typologie ${ }^{16}$. L'ensemble de cette entreprise déborde largement les problèmes que l'on rencontre dans le manuscrit de Munich, mais elle a des conséquences sur le regard que l'on pose ou non sur ces textes. Ces derniers sont pris en compte dans des études consacrées à la transmission et à la circulation des textes sur un temps long et à travers de vastes espaces ${ }^{17}$. Ils sont aussi intégrés dans les recherches consacrées aux étapes permettant de retracer le fil qui mène à une découverte. C'est le cas par exemple de l'étude d'Harald Gropp à propos du problème de « La traversée du loup, de la chèvre et du chou » et de sa présence dans les Propositiones ad acuendos iuvenes. Cet auteur propose une approche comparée impliquant d'autres civilisations ${ }^{18}$. Le recueil attribué à Alcuin, réalisé dans le contexte d'une transformation de l'enseignement, conserve la mémoire de ces problèmes pour l'Occident latin, et a pris une importance particulière aux yeux des historiens. David Singmaster s'est interrogé en 1998 à propos des traditions dont ce texte pouvait être la convergence ${ }^{19}$. En le rattachant à des traditions antiques comme celle d'Euclide

14. Le mot « important » n'est pas explicité, il renvoie peut-être aux problèmes qui lui semblent les plus répandus.

15. Cette initiative s'inscrit dans la continuité d'une école allemande, puisqu'il s'agit d'un quatrième volume, ajouté en 1980, à la réédition de l'œuvre de Johannes Tropfke, érudit du XIX ${ }^{\mathrm{e}}$ siècle : J. TropfKe, Geschichte der Elementarmathematik, vol. 1, Arithmetik und Algebra, éd. K. Vogel, K. ReIch, H. Gericke, Berlin/New York, 1980.

16. On retrouve dans J. TROPFKE (Geschichte der Elementarmathematik...), à côté d'un type abstrait, pseudo-réel, un autre récréatif qui est proche de celui de M. Folkerts.

17. Ainsi, par exemple, en 1983, Kurt Vogel propose de retracer l'histoire des problèmes d'un livre de calcul byzantin du $\mathrm{XIV}^{\mathrm{e}}$ siècle à travers un arbre chronologique et culturel permettant d'observer les héritages sur une échelle de temps très long $\left(\mathrm{V}^{\mathrm{e}}-\mathrm{XV}^{\mathrm{e}}\right.$ siècles) : $\mathrm{K}$. VoGeL, Ein byzantinisches Rechenbuch des frühen 14. Jahrhunderts : Text, Übersetzung und Kommentar, Vienne, 1968, vol. 6, p. 154-159. Quelques années auparavant, il avait proposé avec Herbert Hunger une édition d'un texte grec du $\mathrm{Xv}^{\mathrm{e}}$ siècle d'une centaine de problèmes : Ein byzantinisches Rechenbuch des 15. Jahrhunderts: 100 Aufgaben aus dem Codex Vindobonensis Phil. Gr. 65. Text, Übersetzung und Kommentar, éd. H. Hunger et K. Vogel, Vienne, 1963 (les p. 85 à 101 proposent une mise en relation de ces problèmes avec d'autres textes édités).

18. Voir H. Gropp, « Propositio de lupo et capra et fasciculo cauli. On the History of RiverCrossing Problems », dans Karl der Grosse und sein Nachwirken. 1200 Jahre Kultur und Wissenschaft in Europa, Band II, Mathematisches Wissen, éd. P. L. ButZer, W. OBERsChelP, H. Th. Jongen, Turnhout, 1998, p. 31-41.

19. Voir D. Singmaster, « The History of Some of Alcuin's Propositions », dans Karl der Grosse und sein Nachwirken. 1200 Jahre Kultur und Wissenschaft in Europa, Band II, Mathematisches Wissen..., p. 11-29. 
et de Diophante, à l'Anthologie grecque ou à divers textes chinois, il a tenté de dégager la singularité de l'évènement que ce texte pourrait représenter et des changements qu'il est susceptible d'avoir introduits ${ }^{20}$. Il isole les problèmes des périodes antérieures, qui constituent un héritage possible du texte édité des Propositiones ad acuendos juvenes, de ceux qui ne se trouvent que dans ce texte et qui semblent lui être propres. C'est à une de ces enquêtes qu'invite aussi l'étude d'André Allard publiée en 1998, qui se concentre sur des textes appartenant au même groupe que celui de Curtze ${ }^{21}$.

Récemment, Jacques Sesiano a proposé un volume entier consacré aux Récréations mathématiques au Moyen Âge, qu'il définit à partir de leur énonciation : « [...] au contraire des problèmes d'application usuels, leurs conditions sont peu vraisemblables, voire même absurdes ${ }^{22} »$. Il ne s'agit pas tant de s'intéresser au « peu vraisemblable », qui pourrait renvoyer à des énoncés qui ne présentent pas d'application directe, que de mettre en valeur l'idée d'une gratuité de la démarche. Cette approche récente semble dépasser le simple catalogue pour renouveler en partie, grâce au panorama produit, la compréhension que l'on a de la pensée mathématique d'une époque - comme le Moyen Âge - et de ses dynamiques d'évolution. Si cet ouvrage couvre une très large documentation, il ne considère cependant pas les recueils décrits par Folkerts comme un corpus, mais comme des exemples $^{23}$.

\section{Inscrire ces recueils dans une histoire de l'enseignement universitaire}

Dès 1954, G. Beaujouan propose une lecture de cette production en l'insérant dans le cadre d'une étude sur l'enseignement de l'arithmétique à l'université. Il a ainsi inscrit ces recueils de problèmes dans une perspective historique. Le modèle supportant son raisonnement est l'édition faite par M. Curtze, qu'il compare avec d'autres occurrences extraites de manuscrits

20. L'un des problèmes les plus répandus, introduit par les Propositiones ad acuendos iuvenes, est celui des trois couples d'hommes et de femmes qui doivent traverser une rivière sur une barque pour deux personnes sans qu'un homme ne soit avec une femme différente de la sienne : voir D. Singmaster, « The History of Some of Alcuin's Propositions »... On peut lui associer celui du loup, de la chèvre et du chou, moins représenté mais très connu (analysé par J. Sesiano, Récréations mathématiques au Moyen Âge...., p. 175). Il s'agit des problèmes 17 et 18 des Propositiones (Die älteste mathematische Aufgabensammlung in lateinischer Sprache : die Alkuin zugeschriebenen Propositiones ad acuendos iuvenes..., p. 54).

21. A. Allard, «Quelques problèmes imagés d'arithmétique témoins d'une culture médiévale ", dans J.-C. Polet éd., Patrimoine littéraire européen. Actes du colloque international. Namur, 26, 27 et 28 novembre 1998, Bruxelles, 2000, p. 119-34.

22. Voir J. Sesiano, Récréations mathématiques au Moyen Âge..., p. 3. On trouve dans A. Heefrer, « How Algebra Spoiled Recreational Problems : A Case Study in the CrossCultural Dissemination of Mathematics », Historia Mathematica, 41/4 (2014), p. 400-437, une idée très proche puisqu'il souligne que l'aspect récréatif de ces problèmes vient d'une solution surprenante dont l'utilisation n'est pas évidente pour les énoncés proposés.

23. J. Sesiano, Récréations mathématiques au Moyen Âge..., n. 5, p. 260. 
des bibliothèques françaises et ceux attribués à Alcuin dans la Patrologia latina. Il situe ces cautelae dans le cadre de l'enseignement en expliquant qu'elles « exigent plus d'astuce que de science et évoquent plutôt l'oisiveté de la vie courtoise que le travail universitaire. Pourtant leur utilisation dans les écoles ne saurait être mise en doute ${ }^{24} »$. Pour lui, ce sont des objets construits au tournant des XII et XIII ${ }^{\mathrm{e}}$ siècles, dans la proximité de Joannes de Sacrobosco, si ce n'est par lui-même. De cette façon, l'activité de résolution de problèmes se trouve articulée au texte très diffusé qu'est l'Algorismus (De arte numerandi) de cet auteur parisien, en présupposant que ces problèmes manquaient à ce texte ${ }^{25}$. Mais la coprésence de ces textes ne nous donne en définitive que peu de renseignements sur les liens qu'il pouvait y avoir entre eux à l'origine. Les codices sont souvent composites et il serait nécessaire d'étudier au moins leur composition pour résoudre ce point ${ }^{26}$. G. Beaujouan fonde son idée sur un exemple dont la solution donnée semble relever d'une suite de calculs élémentaires faits sur une table à poussière ${ }^{27}$. Cela le conduit à chercher des variantes afin de repérer des évolutions qui puissent refléter l'introduction de nouveaux savoirs dans la formation universitaire. À cette fin, il met en avant l'influence des Propositiones ad acuendos juvenes attribuées à Alcuin, du De arithmeticis propositionibus du pseudo-Bède, du Liber Abaci de Léonard de Pise (Leonardo Fibonacci) et du Liber augmentis et diminutionis ${ }^{28}$. Le rapprochement proposé entre les recueils repose sur leurs caractères communs et, en particulier, sur le fait qu'ils sont constitués de listes. La correspondance possible d'un ensemble de problèmes s'organise sur deux niveaux : formel (celui de l'énoncé) et mathématique. Il me semble toutefois que le fait de mettre ces textes en relation suppose qu'ils avaient avant tout, dans la tradition, une vocation pédagogique. Pour G. Beaujouan, l'évolution des problèmes, qu'ils contiennent, semble se faire en direction d'une plus grande place accordée à la « courtoisie », ce qu'il déduit en constatant la place donnée à des méthodes de résolution fondées sur des connaissances plus mnémotechniques que mathématiques. Il consacre deux pages à un développement sur l'un de ces vers, qu'il aborde en considérant un corpus plus large, incluant

24. G. Beaujouan, «L'enseignement de l'arithmétique élémentaire à l'Université de Paris... », p. 116.

25. Ibid., p. 122.

26. En l'état actuel de nos connaissances, une dizaine de recueils se trouvent associés dans le même codex avec l'Algorismus de Sacrobosco ou avec le Carmen de algorismo d'Alexandre de Villedieu, ce qui fait environ un tiers.

27. Il s'agit du problème de l'arbre à 12 branches abritant 12 nids dont chacun contient 12 œufs.

28. Sur le Liber augmentis et diminutionis, voir la contribution de M. Moyon dans le présent numéro de Médiévales, «À propos d'algorithmes mathématiques élémentaires dans un corpus de textes arabo-latins du Moyen Âge », p. 57-74. 
notamment un manuscrit français du $\mathrm{Xv}^{\mathrm{e}}$ siècle ${ }^{29}$. Plus récemment, Albrecht Heeffer propose d'établir un rapport entre cette production de recueils et le développement de l'usage de l'algèbre ${ }^{30}$, en montrant que la dimension récréative des problèmes disparaît lorsqu'une méthode plus générale est utilisée ${ }^{31}$.

\section{Évolution et usages des cautelae}

\section{Comment définir et caractériser ce corpus?}

On peut considérer que le terme de « recueil » renvoie à l'idée d'une collection de problèmes ordonnée, dont la succession peut faire sens $^{32}$. Ce qui a au moins deux conséquences sur la documentation considérée (voir Tableau 4 : Les quatre problèmes les plus copiés) : 1) à la différence de M. Folkerts, les recueils ne contenant qu'un seul problème seront systématiquement écartés ${ }^{33}$;2) les proximités identifiées entre les collections conduiront, ensuite, à intégrer de nouveaux recueils au corpus. La comparaison repose sur l'exposé formel des énoncés ou sur des enjeux mathématiques sous-jacents. L'ordonnancement d'un ensemble de problèmes peut former une série que l'on retrouve dans différents exemplaires. Bernard Vitrac a récemment discuté la pertinence de cette approche pour les corpus pseudo-héroniens, en montrant que les emprunts portent très souvent sur des groupes de problèmes, qui semblent donc bien fonctionner en série ${ }^{34}$. Enfin, la langue véhiculaire n'est pas retenue comme

29. La comparaison a été faite à partir du ms. Paris, BnF. fr. 1339. Nous reviendrons plus loin sur la question de la versification.

30. A. HeEFFER, « How Algebra Spoiled Recreational Problems... ».

31. J. SESIANO (Une introduction à l'histoire de l'algèbre..., p. 25) présente une approche un peu différente, puisqu'il propose de voir comment ces problèmes intègrent une partie obligée des ouvrages portant sur l'algèbre. Leur traitement algébrique ne semble pas les rendre obsolètes.

32. Les recueils reflètent cette idée. On a pu voir, dans l'exemple du manuscrit de Munich édité par M. CurTzE («Arithmetische Scherzaufgaben aus dem 14. Jahrhundert » ...), que ces textes utilisent un vocabulaire structurant les relations entre les problèmes. À cela s'ajoute une dimension plus codicologique dans la mesure où le plus souvent les occurrences de problèmes sont introduites par un pied de mouche indiquant un retour à la ligne.

33. M. Folkerts a fait le choix inverse en intégrant à sa liste les mss Oxford, Bodleian Library, Digby 147 ; Oxford, Bodleian Library, Bodley 507 et 496 ; London, British Library, Royal 15 B IX ; London, British Library, Sloane 3281 ; et Erfurt, Ampl. Q.361 (qui n'abrite qu'un seul problème).

34. B. Vitrac, «Collections et séries de problèmes métrologiques dans le corpus héronien et pseudo-héronien », SHS Web of Conferences, 22 (2015), [en ligne] https://doi.org/10.1051/ shsconf/20152200002. Dans cet article, l'auteur propose une discussion intéressante de l'approche décrite dans W. VAN EGMOND, « Types and Traditions of Mathematical Problems : A Challenge for Historians of Mathematics », dans M. FolKerTs éd., Mathematische Probleme im Mittelalter. Der lateinische und arabische Sprachbereich, Wiesbaden, 1996, p. 378-728. 
un critère distinctif, dans la mesure où l'on connaît des exemples de ces textes en langue vernaculaire, notamment en français à partir du $\mathrm{Xv}^{\mathrm{e}}$ siècle ${ }^{35}$.

Soixante et un recueils ont ainsi pu être inventoriés entre le XIII ${ }^{\mathrm{e}}$ et le $\mathrm{Xv}^{\mathrm{e}}$ siècle. Seuls 39 ont actuellement été dépouillés (Tableau 2). Ce corpus n'est pas clos car il n'est pas rare qu'au détour d'un codex émerge une série qui n'a pas été identifiée dans le catalogue lui correspondant. La taille moyenne des recueils, d'après notre corpus, est d'une vingtaine de problèmes, quel que soit le siècle, avec des écarts qui peuvent être assez importants ${ }^{36}$. Les cautelae ont manifestement connu un certain succès. Trois de ces collections ordonnées présentent un nombre très élevé de problèmes au regard de la norme qu'on vient d'établir. Cela reflète la tendance agrégative de ces recueils dont on peut aisément identifier les sources. Les manuscrits London, British Library, Cotton, Cleopatra B IX (XIV siècle), Tours, BM, 399 ( $\mathrm{XV}^{\mathrm{e}}$ siècle) et Oxford, Bodleian Library, Digby 98 ( $\mathrm{XV}^{\mathrm{e}}$ siècle) proposent respectivement 49,41 et 75 problèmes, dont une partie peut être considérée comme une reprise du texte des Propositiones ad acuendos iuvenes ${ }^{37}$. Ainsi, la multiplication des recueils depuis le XIII ${ }^{\mathrm{e}}$ siècle produit un ensemble qui est suffisamment cohérent pour être distingué de la tradition du moine carolingien ${ }^{38}$. Ces collections intègrent donc des traditions dont la circulation mériterait sans doute une analyse plus précise.

35. J'ai pu donner une transcription du recueil contenu dans le ms. Tours, BM, 399 : S. Lamassé, Pour une histoire des problémes mathématiques au Moyen Âge, mémoire de DEA, Université Paris 1 Panthéon-Sorbonne, 1999. Sur le plan de la langue utilisée, notre choix nous conduit à ne pas envisager l'hypothèse, pourtant raisonnable, que le passage d'une langue à l'autre peut produire des effets sur ces cautelae.

36. Ce tableau reflète la dispersion de la série :

\begin{tabular}{rrrrrrr}
\hline min. & max. & écart type & 1e quartile & moyenne & mediane & 3e quartile \\
\hline 3.00 & 49.00 & 11.36 & 15.00 & 20.75 & 21.50 & 25.50 \\
4.00 & 75.00 & 15.42 & 17.25 & 24.61 & 23.50 & 27.50 \\
\hline
\end{tabular}

Vingt-six recueils présentent des collections dont la quantité de problèmes varie dans un intervalle entre dix et trente items.

37. Ces recueils ont déjà été identifiés dans M. FolKerTs, « Mathematische Aufgabensammlungen aus dem ausgehenden Mittelalter »..., où l'auteur signale par exemple que le ms. London, British Library, Cotton, Cleopatra B IX présente 12 problèmes attribués à Alcuin, mais ce qui nous semble intéressant, c'est l'organisation d'une dizaine d'entre eux en série aux f. 19r-20r, d'autres sont identifiables mais sont plus épars dans le texte. On retrouve aussi, en série, des extraits du texte attribué à Alcuin dans le ms. Tours, BM, 399. Le ms. Oxford, Bodleian Library, Digby 98 comprend 40 problèmes de cet héritage structurés en série.

38. Si l'on observe la production du texte tel qu'on peut la percevoir au travers de l'édition des Propositiones ad acuendos iuvenes attribués à Alcuin (Die älteste mathematische Aufgabensammlung in lateinischer Sprache : die Alkuin zugeschriebenen Propositiones ad acuendos iuvenes...). Quatorze manuscrits ont été utilisés pour cette édition et 10 d'entre eux ont été produits avant le $\mathrm{XI}^{\mathrm{e}}$ siècle, leur quantité chute après alors qu'au $\mathrm{XIII}^{\mathrm{e}}$ siècle. $A$ contrario, les recueils se développent à un point tel que l'on peut se demander s'il n'y a pas un remplaçant des Propositiones. 


\begin{tabular}{|c|c|c|c|}
\hline & Localisation & Référence & Folios \\
\hline $\mathrm{x}$ & Barcelone & Biblioteca Central de Catalunya, 39 & $54 v-57 \mathrm{r}$ \\
\hline $\mathrm{x}$ & Berlin & Staatsbibliothek lat. oct. 259 & $83 v-85 r$ \\
\hline \multirow[t]{2}{*}{$\mathrm{x}$} & Berlin & Staatsbibliothek lat. quart. 485 & p. 22-24 \\
\hline & Berlin & Staatsbibliothek zu Berlin, Ms. lat. qu. 846 & $9 \mathrm{r}-10 \mathrm{v} ; 9 \mathrm{v}-10 \mathrm{v}$ \\
\hline \multirow[t]{3}{*}{$\mathrm{X}$} & Berlin & Staatsbibliothek, lat. quart. 587 & $\begin{array}{l}119 \mathrm{r}-122 \mathrm{v} ; 171 ; 182 \mathrm{r}- \\
193 \mathrm{v} ; 192 \mathrm{v}-194 ;\end{array}$ \\
\hline & Bologne & Biblioteca Universitaria 164 & $56 \mathrm{v}-58 \mathrm{r} ; 56 \mathrm{v}-57 \mathrm{v}$ \\
\hline & Boston & The Francis A. Countway Library of Medicine, 20 & $174 \mathrm{r}-178 \mathrm{r} ; 174 \mathrm{r}-178 \mathrm{v}$ \\
\hline $\mathrm{x}$ & Bâle & Universitätsbibliothek, O.IV.37 & $58 \mathrm{v}-60 \mathrm{v}$ \\
\hline $\mathrm{x}$ & Cambridge & Trinity College O.2.45 & $32 \mathrm{r}-36 \mathrm{v}$ \\
\hline $\mathrm{x}$ & Colmar & BM 365 & $9 \mathrm{r}-10 \mathrm{r}$ \\
\hline $\mathrm{x}$ & Erfurt & Ampl. Q. 343 & $92 r-94 v$ \\
\hline $\mathrm{x}$ & Erfurt & Ampl. Q. 345 & $16 r-16 v$ \\
\hline \multirow[t]{2}{*}{$\mathrm{x}$} & Erfurt & Ampl. Q. 345 & 61 \\
\hline & Erfurt & Ampl. Q. 351 & $99 \mathrm{a}-\mathrm{b}$ \\
\hline \multirow[t]{2}{*}{$\mathrm{x}$} & Erfurt & Ampl. Q. 368 & $148 \mathrm{v} ; 154 \mathrm{v}$ \\
\hline & Erlangen & Universitätsbibliothek, 664 & $73 v-74$ \\
\hline $\mathrm{x}$ & Glasgow & Hunter 461 & $130 \mathrm{v}-133 \mathrm{r}$ \\
\hline $\mathrm{x}$ & Londres & Addit. 25031 & $12 \mathrm{v}-13 \mathrm{r}$ \\
\hline \multirow[t]{2}{*}{$\mathrm{x}$} & Londres & Addit. 30380 & $133 \mathrm{v}-137 \mathrm{r}$ \\
\hline & Londres & British Museum Egerton 2622 & 166 \\
\hline $\mathrm{x}$ & Londres & British Museum, Sloane 513 & $48 \mathrm{r}-51 \mathrm{v}$ \\
\hline $\mathrm{x}$ & Londres & Cotton Cleop. B IX & $17 \mathrm{v}-21 \mathrm{r}$ \\
\hline $\mathrm{x}$ & Londres & Royal 12 C XII & $8 r-11 r$ \\
\hline $\mathrm{x}$ & Londres & Royal 12 E XXV & $183 \mathrm{v}$ \\
\hline $\mathrm{x}$ & Londres & Royal 12 F XIX & $184 r-185 r$ \\
\hline \multirow[t]{4}{*}{$\mathrm{X}$} & Londres & Royal 15 B IX & $78 \mathrm{v}$ \\
\hline & Londres & Society of Antiquaries of London, 306 & $55 v-57 v$ \\
\hline & Mayence & Stadtbibliothek, I 530 a & $29 \mathrm{v}$ \\
\hline & Melk & Stiftsbibliothek, 278 & $23-26$ \\
\hline \multirow[t]{2}{*}{$\mathrm{x}$} & Montpellier & Bibliothèque de la faculté de Médicine, 323 & $235 \mathrm{va}-237 \mathrm{vb}$ \\
\hline & Munich & $\operatorname{Cgm} 739$ & $44 r-45 r$ \\
\hline $\mathrm{x}$ & Munich & Clm 14684 & $30 \mathrm{r}-33 \mathrm{r}$ \\
\hline $\mathrm{x}$ & Munich & Clm 18460 & $4 \mathrm{r}$ \\
\hline $\mathrm{x}$ & Munich & $\mathrm{Clm} 534$ & $36 \mathrm{r}-37 \mathrm{v}$ \\
\hline $\mathrm{x}$ & Munich & Clm 5963 & $47 v-48 v$ \\
\hline \multirow[t]{2}{*}{$\mathrm{x}$} & Munich & Clm 8951 & $44 v-47 v$ \\
\hline & Oxford & Bodl., Lyell 36 & $18-19 v$ \\
\hline $\mathrm{x}$ & Oxford & Digby 104 & $72 v-74 r$ \\
\hline $\mathrm{x}$ & Oxford & Digby 193 & $25 v-26 v$ \\
\hline $\mathrm{x}$ & Oxford & Digby 75 & $132 \mathrm{r}-135 \mathrm{v}$ \\
\hline $\mathrm{x}$ & Oxford & Digby 98 & $34 r-39 v$ \\
\hline \multirow[t]{2}{*}{$\mathrm{x}$} & Paris & Bnf lat. 14070 & $92 \mathrm{v}-93 \mathrm{r}$ \\
\hline & & Bnf lat. 14070 & $94 v-96 r$ \\
\hline $\mathrm{x}$ & Paris & Bnf lat. 16089 & $86 r-91 v$ \\
\hline \multirow[t]{2}{*}{$\mathrm{x}$} & Paris & Bnf lat. 7196 & $7 \mathrm{r}, 7 \mathrm{v}, 31 \mathrm{r}$ \\
\hline & Paris & Bnf lat. $7420 \mathrm{a}$ & \\
\hline $\mathrm{x}$ & Paris & Bnf lat. 7475 & $119 \mathrm{r}-123 \mathrm{r}$ \\
\hline \multirow[t]{2}{*}{$\mathrm{x}$} & Paris & Bnf. Nouv. acq. lat. 657 & $60 v-61 v$ \\
\hline & Prague & Knihovna Metropolitni kapitulní, M.CIII.(1463) & $205 \mathrm{v} ; 207 \mathrm{r}-208 \mathrm{r}$ \\
\hline \multirow[t]{2}{*}{$\mathrm{x}$} & Reims & 1101 & \\
\hline & Tournai & Bibliothèque de la Ville, Cod. 87 & $122 \mathrm{ra}-128 \mathrm{vb}$ \\
\hline \multirow[t]{10}{*}{$\mathrm{x}$} & Tours & Tours 399 & \\
\hline & Uppsala & Uppsala, C7 & $118 \mathrm{r}$ \\
\hline & Vatican & Vat.lat. 3127 & $89-92$ \\
\hline & Verdun & BM 25 & \\
\hline & Vienne & Vienne 3502 & $222 r-223 r$ \\
\hline & Vienne & Vienne 4987 & $93 v-96 v$ \\
\hline & Vienne & Vienne 5166 & 159 \\
\hline & Wolfenbüttel & Herzog August Bibliothek 16.1 Astronom. 4 & $69 v-70 v$ \\
\hline & Wolfenbüttel & Herzog August Bibliothek, Cod. Guelf. 17.21. Aug. quart. & $60 \mathrm{r}-\mathrm{v} ; 60 \mathrm{r}-\mathrm{v}, 85 \mathrm{r}-\mathrm{v}$ \\
\hline & Wolfenbüttel & Herzog August Bibliothek, Cod. Guelf. 696. Helmst. & $52 \mathrm{v}$ \\
\hline
\end{tabular}

Tableau 2 : Manuscrits contenant des cautelae ayant été dépouillés. 


\section{Organisation et homogénéité des recueils}

L'utilisation de la typologie légèrement modifiée de M. Folkerts ${ }^{39}$ permet de comparer les recueils sous la forme d'alignements de séquences ordonnées de problèmes (Tableau 3). Leurs représentations sous forme de tapis de séquences ${ }^{40}$ reflètent des situations assez différentes pour les deux derniers siècles du Moyen Âge. Le XIV siècle semble marqué par deux phénomènes. Celui des variantes, tout d'abord, qui apparaissent regroupées au moins pour le début des recueils. Ces dernières concernent les « problèmes indéterminés », les «nombres pensés et objets cachés » et dans une moindre mesure la rubrique « distribution et partage ${ }^{41}$. Le second phénomène apparaît si l'on fait abstraction du précédent, on discerne sur les premiers problèmes une succession de quatre grands thèmes $(2,1,4,7)$. Pourtant, au-delà de ces quelques traces, ces séquences ne semblent pas vraiment se fixer autour d'un schéma commun, en tout cas pas dans l'état de notre dépouillement. Une approche par séquences thématiques conduit cependant à minorer la question de la circulation des problèmes. Or, plus d'une dizaine d'entre eux sont présents dans plus de la moitié des recueils. Certains, amplement recopiés, ont pu devenir de véritables lieux communs $(\text { Tableau } 4)^{42}$ :

39. M. FOLKERTS, " Mathematische Aufgabensammlungen aus dem ausgehenden Mittelalter »... Voir la présentation de la typologie (Tableau 1. La typologie.....). Nous préférons utiliser certaines dénominations de J. Sesiano qui nous semblent moins restrictives : « Nombres pensés et objets cachés » pour « deviner les chiffres » et « Distribution et partage » pour « Le gardien dans le jardin ». Utiliser la typologie de M. Folkerts nécessite d'associer des problèmes à des catégories par extension, mais un certain nombre d'entre eux ne peuvent pas y trouver place, comme ceux qui concernent la géométrie.

40. Nous avons utilisé la bibliothèque TramineR du logiciel de statistique $\mathrm{R}$ [en ligne] http://traminer.unige.ch/documentation.shtml [consulté le 17 juillet 2019]. Avec un effectif aussi faible que celui que nous considérons, il n'est pas si facile de se faire une idée précise du recueil type. Dans ce type de graphique, les ordonnées donnent l'ordre de la suite des problèmes du premier jusqu'au $n^{\text {ième }}$ et chaque ligne d'abscisse désigne les recueils. Les blancs regroupent des problèmes qui ne sont pas explicitement définis dans la typologie de M. Folkerts ou qui appartiennent explicitement à l'ensemble des Propositiones ad acuendos iuvenes.

41. Il arrive que ces variantes soient souvent signalées dans le fil des recueils. On peut ainsi observer dans un manuscrit du $\mathrm{xv}^{\mathrm{e}}$ siècle une distinction entre les « idem » qui indiquent des variantes et les numéros $« 1^{\mathrm{m}} », \ll 2^{\mathrm{m}} »$ qu'il indique dans la marge. Voir le ms. Basel, Universitätsbibliothek, O. IV. 37, f. 58v-60v.

42. Dans la thématique 2 de M. Folkerts et débutant tout un ensemble de séquence du $\mathrm{XIV}^{\mathrm{e}}$ siècle, le problème le plus diffusé est sans aucun doute : " [S]int hic milites, pedites et puelle et sint in universo 12 et habeant 12 panes inter se parciendos, et quilibet miles capiat IIos panes, quelibet puella medietatem panis quibus pedes quartam partem panis. Queritur ergo quot erunt milites, quot pedites, quot puelle ? (Paris, BnF, lat. 7196, f. 7rb). 
Tableau 3 : Les séquences de problèmes aux $\mathrm{XIV}^{\mathrm{e}}$ et $\mathrm{XV}^{\mathrm{e}}$ siècles.
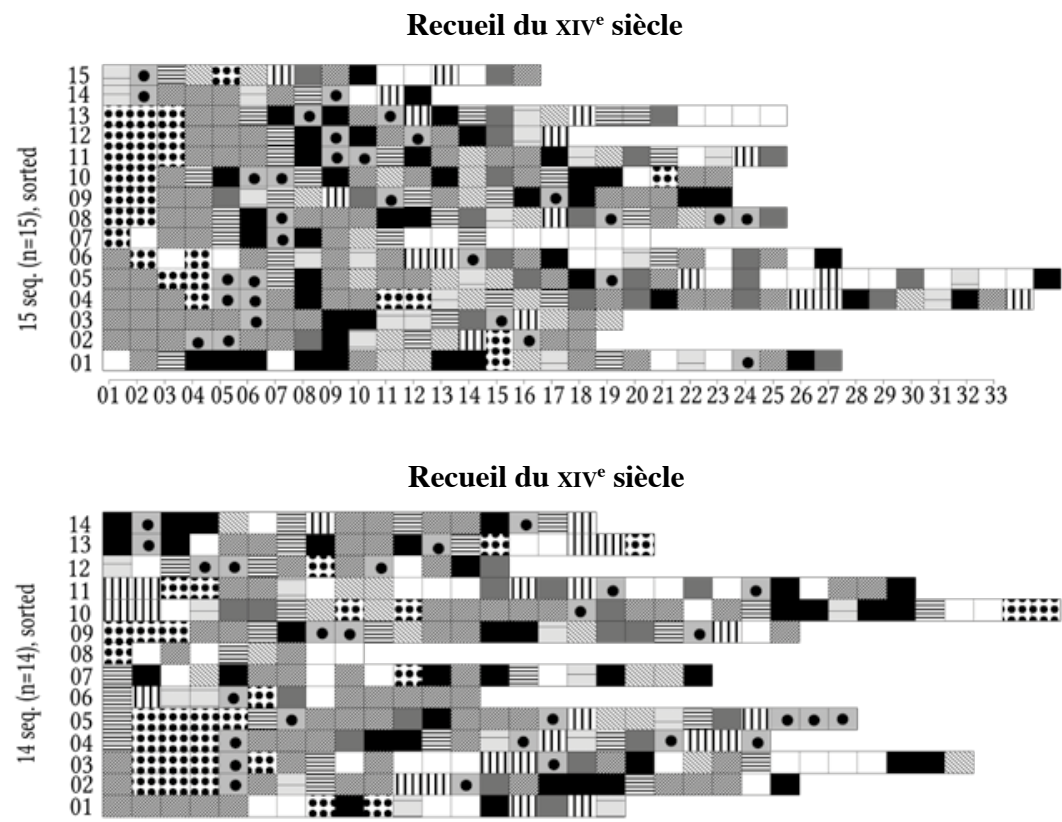

010203040506070809101112131415161718192021222324252627282930313233

$\square$ Différent de la typologie
$\square$ Distribution et partage
$\square$ Nombre pensés et objets cachés
目 Prendre et donner
․ Problème de convivialité (indéterminés)
$\square$ Problème de transport
$\square$ Problème linéaire à une inconnue
$\square$ Problèmes plaisants
$\square$ Transvasements

Tableau 4 : Les quatre problèmes les plus copiés

\begin{tabular}{|c|c|c|}
\hline Type & Thème & Nombre de copies \\
\hline 2 & Couples de jaloux & 22 \\
\hline 1 & Cacher quelque-chose sans sa main & 21 \\
\hline 6 & Les offrandes dans une église & 19 \\
\hline 9 & Distribuer du vin & 18 \\
\hline
\end{tabular}


On constate donc une assez grande homogénéité ${ }^{43}$, avec un groupe de 22 recueils dont 24 problèmes ont de très fortes proximités, ce qui offre une vision différente de l'approche thématique. L'un des effets de cette densité est de témoigner des éléments d'une culture commune pour les lecteurs de ces documents. Pour l'historien d'aujourd'hui, cela permet de mieux suivre certains héritages, comme celui d'Alcuin dont on peut percevoir l'influence à travers des séries, mais aussi à travers des problèmes singuliers. Les solutions proposées renforcent enfin la cohérence de ces recueils par les mises en œuvre mathématiques et logiques qu'ils supposent ${ }^{44}$. Ces dernières se déroulent sous forme de recettes, dépourvues de justifications. Par exemple, la résolution du problème indéterminé de la distribution de pain se contente d'en fournir le résultat : « Sola puella manet / peditum sex / quinque quirites » (« Il ne reste qu'une seule fille, six fantassins et cinq citoyens »). On ne propose donc pas de calcul, mais l'efficacité du jeu suppose une activité de vérification du résultat nécessitant une certaine adresse afin de manier les deux fractions simples proposées ${ }^{45}$. Les solutions ne dévoilent, le plus souvent, rien d'autre qu'un mécanisme dans lequel on ne perçoit que quelques traces de connaissances mathématiques. Par exemple, certaines devinettes mobilisent pour leur résolution les notions de pairs et d'impairs qui sont un des fondements de la division du nombre au Moyen Âge ${ }^{46}$. Souvent les méthodes exposées supposent un certain labeur calculatoire comme dans le problème de l'échiquier, où l'on propose un algorithme de résolution, ou bien encore dans celui des dons faits à l'église (type 6). Mais l'activité mathématique proposée peut présenter un certain intérêt en matière de raisonnement. Prenons par exemple le problème des trois hommes et trois femmes formant un couple que l'énonciateur se propose ici de révéler :

Des trois hommes et des femmes, dont tu veux identifier laquelle est la femme de qui. Donne une unité à la première, à l'autre 2, à la troisième 3. Puis, au mari d'une femme note le double de son argent, au mari de la

43. Il est possible de mesurer ces relations afin de classer les collections. On peut, pour cela, produire une matrice d'adjacence et utiliser un indice de liaison décrit dans A. GUERREAU et M.-A. Polo de Beaulieu, « Classement des manuscrits et analyse factorielles. Le cas de la Scala coeli de Jean Gobi », Bibliothèque de l'École des chartes, 154 (1996), p. 359-400. Ce résultat a été produit et complété d'une analyse factorielle accompagnée d'une classification hiérarchique qui doit permettre d'extraire ces différents groupes.

44. Pour toutes les formalisations mathématiques que posent ces problèmes, voir J. Sesiano, Récréations mathématiques au Moyen Âge..., p. 138-141.

45. On retrouve cette possible activité de vérification dans des problèmes que l'on pourrait formaliser aujourd'hui comme des systèmes d'équations à deux inconnus caractéristiques du type « Donner et prendre ».

46. Pour un aperçu général on se référera à J. Sesiano, Récréations mathématiques au Moyen Âge..., p. 239 sq. Le texte le plus emblématique du Moyen Âge est sans aucun doute le De institutione arithmetica de Boèce qui débute par la division des nombres en pairs et impairs, on retrouve ces idées dans l'apprentissage élémentaire comme l'atteste le Carmen de Algorismo d'Alexandre de Villedieu. 
suivante multiplie son argent par 9, et pour le troisième multiplier par 10 . Après cela collecte tous les nombres et cherche le complément pour avoir 60 , et de ce complément fait ressortir les $8^{\mathrm{e}}$ qui donne le nom de celui qui a doublé et l'unité est le nom de celui multiplié par 9. Ces deux-là ayant été trouvés, le troisième est alors évident ${ }^{47}$.

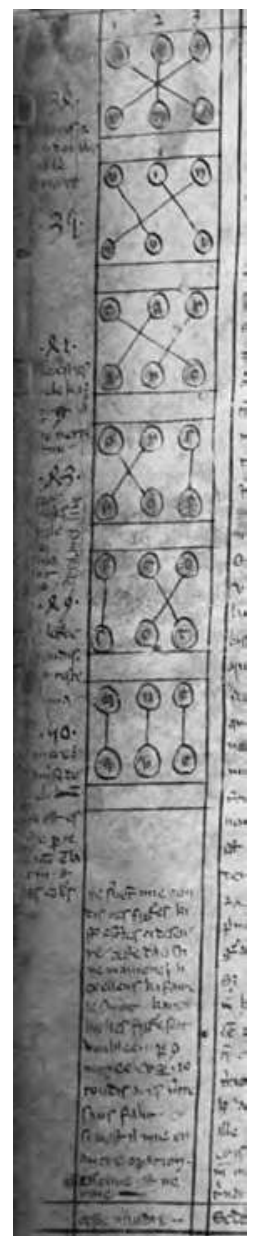

G. Beaujouan remarque et analyse l'utilisation de phrases en guise de solution. Il s'appuie sur l'exemple suivant $^{48}$ : " Arnoldus bigamum, katulos, erumpgna, fugavit glutinat ». Il s'agit de faire correspondre à l'ordre des voyelles alphabétiques les valeurs 1, 2, 3 . Dans «Arnoldus », A O U se suivent dans l'ordre que ces lettres occupent dans l'alphabet. On leur attribue donc les valeurs 1, 2, 3. Mais pour «Katulos », A U O donnera $1,3,2$, car $\mathrm{U}$ se situe après $\mathrm{O}$ dans l'alphabet, alors que dans ce contexte il est placé avant. Ce qu'il faut retenir de ces mots, ce n'est pas qu'ils proposent une solution, mais toutes les solutions : $(1,2,3),(2,1,3)$, $(1,3,2),(2,3,1),(3,1,2),(3,2,1)$. Une autre représentation confirme cette hypothèse (Figure 1). Elle est contenue dans le ms. London, British Library, Sloane 3281 (fin du XIII ${ }^{\mathrm{e}}$ siècle) :

Ce sont les différentes combinaisons possibles qui sont représentées dans les six cases. À chaque fois, des ronds entourant une lettre sont reliés par des traits figurant la relation entre les hommes et les femmes. Les nombres écrits sur la gauche avec le texte qui les accompagne reflètent les mêmes opérations arithmétiques que celles présentes dans le manuscrit utilisé par G. Beaujouan. Ainsi, les solutions, qu'elles se présentent sous une forme de suite de mots ou d'un ensemble de graphiques permettent de mémoriser

Figure 1 : Réunir les couples

(ms. London, British Library, Sloane 3281, f. 26r (C) The British Library Board).

47. «Si de tribus hominibus et eorum uxoribus scire volueris, que illarum cuius uxor fuerit, detur uni unitas in. censii, alie dentur 2, tercie 3. Deinde maritus unius notate mulieris duplet censum suum, maritus alterius notate multiplicet censum suum per 9, tercius eciam suum per 10 multiplicet. Postea colligatur totalis numerus et queratur, per quot possunt complere 60 , et in illius complecionis octonariis exibit nomen duplati, in unitatibus vero nomen per 9 multiplicati. Habito igitur de duobus, de tercio manifestum est » (éd. M. CurTZE, « Arithmetische Scherzaufgaben aus dem 14. Jahrhundert ».., p. 77-88, problème XIX). Dans cet exemple, le copiste développe une solution.

48. G. Beaujouan, « L'enseignement de l'arithmétique élémentaire à l'Université de Paris.... » ;. Sesiano, Récréations mathématiques au Moyen Âge... 
facilement tous les appariements possibles (Figure 1). Et c'est, sans doute, cette agilité-là, qui est recherchée dans ce problème.

Ces recueils sont davantage qu'une tradition textuelle marginale. Ils font circuler de nombreux problèmes identiques et mobilisent des compétences de raisonnement et de calcul. Ce corpus participe très vraisemblablement à une forme de culture mathématique. Il est en outre en constante évolution. On perçoit au XIV ${ }^{\mathrm{e}}$ siècle une structure : une série de problèmes constitue presque un modèle, auquel on peut ajouter des variantes. $\mathrm{Au} \mathrm{XV}$ siècle, en revanche, les cautelae suivent des schémas différents et on $\mathrm{y}$ intègre un nombre important de « nouveaux » problèmes. Cette tradition ne s'est donc pas figée. Elle continue à regrouper et à reprendre, parfois, certaines séries. Cela nous amène à nous interroger sur la place qu'occupent les cautelae dans la culture mathématique.

\section{Les recueils peuvent-ils être considérés comme des éléments de la culture mathématique médiévale?}

Des recueils en contexte arithmétique

La mise en relation de ces recueils avec les Algorismes $^{49}$ est une des tendances que l'on peut observer dans la composition de ces collections. Dès le $X{ }^{\mathrm{e}}$ siècle, on regroupe certains problèmes sous l'intitulé d'opérations que l'on rencontre à la même période pour les Algorismes. C'est ce que l'on trouve dans les manuscrits London, British Library, Additional $30380^{50}$ et Oxford, Bodleian Library, Digby $104^{51}$. Parfois, les indications sont intégrées à la solution, comme peut le faire le manuscrit Bodleian Library, Digby 75, qui, dans un problème de poursuite, propose de faire usage de progressions (f. 134v). Il arrive aussi que l'on enchevêtre des séries appartenant au modèle des cautelae à d'autres traditions de traités, comme c'est le cas dans le manuscrit London, British Library, Sloane $3281^{52}$. De la même façon, la

49. Nous regroupons sous cette désignation générale l'ensemble des traités universitaires portant la dénomination Algorismus, et plus particulièrement ceux de Johannes de Sacrobosco et d'Alexandre de Villedieu.

50. C'est un ouvrage de belle facture contenant la Cautela in addicione (f. 133va), la Cautela substractione (f. 133vb), la Cautela mediacioni, problème du type « donner et prendre » qui consiste à découvrir la somme d'argent que quelqu'un a dans sa bourse, et la Cautela duplacionis (f. 133vb), qui porte sur le problème des dons faits à chaque autel d'une église. Les titres visent à décrire l'opération considérée comme essentielle pour la résolution et sont influencés par l'Algorismus de Sacrobosco.

51. Sur les deux feuillets $72 \mathrm{r}$ et $73 \mathrm{r}$, on remarque à l'encre rouge : Super duplacionem et mediacionem (f. 72r), De multiplicionem (f. 72v). Ce texte signale les problèmes à la suite de ces titres par « Casus », « Item casus », « Item questio », et les résolutions y sont développées. Enfin le texte se termine par un développement sur les racines.

52. Ce recueil assez complexe mériterait plus d'attention. Deux attestations de ces problèmes sont dans ce codex aux f. 26r - déjà repéré par M. Folkerts - et 49r-55v, qui 
série de problèmes proposée dans le Paris, Bnf. 7420a (f. 52v-53v) se situe dans la continuité du Carmen de algorismo d'Alexandre de Villedieu et d'un développement sur le calcul. Les six opérations fondamentales, ou espèces (addition, soustraction, médiation, duplation, multiplication, division) ${ }^{53}$, sont pratiquement toujours utilisées dans le corpus, et les mentions de nombres pairs et impairs laissent penser que l'on peut trouver assez rapidement des relations implicites avec une culture mathématique médiévale élémentaire. Que ces recueils aient un caractère léger ou ludique, les énoncés en témoignent ; mais le fait qu'ils aient été l'objet d'une lecture mathématique liée, entre autres, aux Algorismes médiévaux, les inscrit davantage dans une culture mathématique universitaire.

Le manuscrit Paris, BnF, lat. 14070 (XIV siècle) témoigne, d'une façon qui nous paraît signifiante, de l'usage que l'on a pu faire de ces problèmes. Tout d'abord à travers leur dénomination, dans la mesure où le scripteur substitue à une énonciation complète une autre, plus ramassée, qui n'est pas sans évoquer une forme de locution figée ${ }^{54}$. Par exemple, De tribus sociis et eorum uxoribus pour désigner le problème des 3 hommes dont on ne sait pas quelle est leur femme, ou De numerus habitis in bursa (f. 92v-93v). Il y a là une forme de réification, qui leur donne une dimension plus générale. Les énoncés peuvent être disposés en outre de manière différente. Par exemple, 3 problèmes de devinettes, très diffusés dans le corpus, sont placés dans des cercles à la fin de la série, produisant ainsi un effet de séparation (f. $93 \mathrm{v})^{55}$. Ce manuscrit ajoute enfin un autre recueil débutant par " Perquires verum », qui propose une série de 18 problèmes, dont les premiers font l'objet d'un développement. Le souci de maîtrise des énoncés et de leur solution est lisible dans la construction d'une variante à l'un des problèmes ${ }^{56}$. C'est à partir d'un

nous préoccupe ici. Il débute au f. 49r avec un titre à l'encre rouge : Hic incipiunt noticie algorismi et l'incipit est « Omnia que a primavera... »; il présente ensuite la numération et les opérations élémentaires, introduit les premiers problèmes au f. 50r à la fin de la soustraction, débute un groupe de problème par "Sint hic milites, pedites et puelle » jusqu'au f. 51r et reprend au $\mathrm{f}$. $54 \mathrm{r}$ avec «Tres fratres sunt habuntes sororem nubilem habencium ». Il y a là le calcul de la quantité d'œufs nécessaires pour un monastère et le nombre de pommes sur des branches. Puis alternent des séries de problèmes identiques à celles des recueils (f. 50ra$50 \mathrm{vb} ; 53 \mathrm{rb}-54 \mathrm{ra}$ ) et d'autres problèmes davantage liés au comput (f. 50vb-53rb ; 54ra-55va). Le texte s'achève au f. 55v par « explicit cauteli algorismi».

53. «Médiation » et « duplacion » consistent à diviser et à multiplier par 2. Ces opérations ont un intérêt pour le calcul mental et sont sans doute les restes d'un héritage des ouvrages de calcul arabes.

54. L'étude de Y. Gentilhomme, "Contribution à une réflexion sur les locutions mathématiques », Cahiers de lexicologie, 66 (1995), p. 5-37, développe notamment l'idée d'un usage de phrasèmes dans la langue mathématique.

55. Successivement, le problème consistant à deviner où se trouvent les choses cachées dans les mains, celui du nombre de deniers que quelqu'un possède dans sa bourse et de l'homme qui donne à son fils une somme pour vivre.

56. L'auteur de ce texte propose la résolution du problème de la hauteur d'une tour dont on sait que le tiers est dans la terre, la moitié dans l'eau et 9 pieds au-dessus de la terre. L'auteur souligne qu'il faut multiplier $6 \times 9$, puis il ajoute une variante : « Si autem vellos 
de ces exemples ${ }^{57}$ que G. Beaujouan a montré comment la solution de ces problèmes pouvait être lue dans la perspective d'une pratique mathématique liée aux connaissances apprises en étudiant les Algorismes à l'aide d'une table (f. 95r).

\section{Hors des recueils}

L'utilisation de ces problèmes dans d'autres traités ou commentaires est assez ancien, puisque dès le XIII ${ }^{\mathrm{e}}$ siècle on connaît un texte nommé $D e$ regulis generalibus algorismi ad solvendum omnes questiones propositas (Règle générale d'algorismes pour résoudre tous les problèmes proposés $)^{58}$. Le manuscrit Oxford, Bodleian Library, Digby 147 (f. 86-88v), utilisé par M. Folkerts, contient ce traité caché sous le titre de Cautele algorismi (« Incipiunt cautele algorismi »). Il s'agit là d'une variante du titre daté du $\mathrm{XIII}^{\mathrm{e}}$ siècle, retenu par Barnabas B. Hughes à partir de six manuscrits. Dans le texte contenu dans ce manuscrit d'Oxford, une dizaine de problèmes sont regroupés sous différents titres et introduits par une règle succincte ${ }^{59}$. Comme le souligne B. B. Hughes, il s'agit moins d'offrir une analyse théorique que de fournir des outils pour le praticien. Les premiers problèmes ont un caractère plus abstrait et ne semblent pas avoir été extraits du corpus des recueils que nous avons analysé. En revanche, ceux qui constituent la suite du texte en sont manifestement repris et ont été choisis et réorganisés autour d'une logique, que nous avons déjà observée, et qui est plus calculatoire ${ }^{60}$. On ne trouve pas dans ces textes, par exemple, de devinettes (type 1 de $\mathrm{M}$. Folkerts). Parmi les règles proposées, certaines sont déjà bien connues à la même époque. En particulier, pour le calcul de l'âge d'un homme, l'algorithme utilisé peut parfaitement être associé à la méthode de simple fausse position. À la fin de ces règles, on en trouve une qui est destinée à l'un des problèmes indéterminés les plus célèbres du corpus. Il s'agit du problème de la distribution d'une quantité de pain à des militaires, à des demoiselles et à des fantassins. Or, on ne donne jamais de

variare istam cautelam tu potes quere quot sunt pedes extra » (f. 95r). Cette fois, on ne connaît pas la longueur de la tour hors de l'eau, il s'agit de retrouver les 9 pieds. L'opération est donc ici une division.

57. Il s'agit de l'exercice signalé note 27 .

58. Texte édité par B. B. Hughes, « De regulis generalibus : A 13th-Century English Mathematical Tract on Problem Solving », Viator, 11 (1980), p. 209-224.

59. Le plan du ms. Oxford, Bodleain Library, Digby 147 est identique à l'édition de B. B. Hugues, «De regulis generalibus... ». On y trouve successivement la Regula de partibus inveniendis per totum, la Regula de toto inveniendo per residuum, la Regula de partibus inveniendis per residuum, la Regula de duplatione vel triplatione et la Regula Tribus quantitativus notis quartum ignotum perscrutari.

60. On y trouve, par exemple, les problèmes portant sur l'âge d'un homme, la longueur d'une lance, les offrandes dans une église, une fuite par 3 broches, un partage de pains entre 3 catégories de personnes. 
solution développée à ce problème ${ }^{61}$. La méthode qu'offre l'auteur anonyme est plutôt une recette. Celle-ci est traduite, dans des versions françaises du $\mathrm{XV}^{\mathrm{e}}$ siècle, sous un autre intitulé, celui d' « Apposition et remotion » (Tableau $5)^{62}$ :

Ils sont ici des hommes d'armes, des fantassins et des jeunes femmes qui sont en tout 20 ou 13 , et on leur distribue 20 pains de telle façon que les hommes d'armes aient deux pains, deux jeunes femmes aient un pain et 4 fantassins un pain. On cherche combien il y a de cavaliers, de fantassins et de jeunes femmes.

Pour résoudre ce type de questions et toutes les similaires, on prend le nombre le plus petit formé [à partir de l'énoncé], que l'on nomme la racine, et qui dans cet exemple est 5 .

En effet, 2 hommes d'armes et 2 fantassins, et une fille ont 5 pains. Et ainsi 5 hommes ont 5 pains. Il faut former ta question ainsi en divisant le nombre entier connu, à savoir ici 20, par la racine, et l'on obtiendra 4 par lequel tu multiplieras par le nombre racine de chaque individu et tu as 8 hommes d'armes, 8 fantassins et 4 jeunes femmes.

Et ainsi 20 n'auront pas plus de 20 pain, comme il est demandé.

$$
\begin{aligned}
x+y+z & =20(1) \\
2 x+\frac{1}{2} y+\frac{1}{4} z & =20(2)
\end{aligned}
$$

On cherche le plus petit entier pour (2), ici c'est 5

$(2,1,2)$ car,

$2 \times(2)+1 \times\left(\frac{1}{2}\right)+2 \times\left(\frac{1}{4}\right)=5$

$8+4+8=20$

Tableau 5 : Exemple du texte édité dans B. B. Hughes, « De regulis generalibus : A 13th-Century English Mathematical Tract on Problem Solving », Viator, 11 (1980), p. 224.

Si l'on examine les ouvrages en langue française relevant d'une tout autre tradition - celle des arithmétiques commerciales -, réalisés à la fin de cette période, ces séries sont présentes, mais sous forme de morceaux

61. Dans la marge du ms. Oxford, Bodleian Library, Digby 147 , on trouve un index sous une forme humaine et cornue. S'agit-il de soulever la difficulté particulière de ce problème ?

62. P. Benoit et S. Lamassé, «Apposition et remotion : une spécificité française », dans M. Spiesser et M. Guillemot éd., De la Chine à l'Occitanie, chemins entre arithmétique et algèbre, Toulouse, 2003, p. 1-13. Voici la version latine du problème contenu dans le Tableau 5 : «Sunt hic milites pedites et puelle in uniuerso 20 uel 13, et distribuas is tis 20,20 panes ita scilicet; quod miles habeat duos panes, due puelle unum panem, 4 pedites unum panem. Queritur quot sunt milites, quot pedites, quot puelle. Ad istam questionem soluendam et omnes consimiles inueniendas, unum minimum numerum formatum ad modum questionis quod vocetur radix ut est 5 in hoc exemplo. Nam 2 milites et 2 pedites et una puella habunt 5 panes. Et sic 5 homines habunt 5 panes. Ad modum forme questionis per illam radicem divide numerum integrorum percipiendorum, scilicet 20, et erit quociens 4 per quod multiplicabis numerum cuiuslibet indiuidui in radice, et sic habes 8 milites et 8 pedites et 4 puelle. Et sic 20 nec habunt inter se plusquam 20 panes, quod est propositum. » Ce problème, résolu de cette façon, se trouve en français dans le ms. Paris, BnF, fr. 1339, f. 73r. 
largement reclassés ${ }^{63}$. Ces opérations d'appropriation et de reclassement de cet héritage ne sont pourtant pas aussi neuves qu'on pourrait le penser, puisqu'une démarche proche se rencontre dans le Liber Abaci de Léonard de Pise. Une des différences que l'on peut noter à propos des textes du $\mathrm{xv}^{\mathrm{e}}$ siècle tient justement à la place qu'une part d'entre eux donnent à l'activité mathématico-ludique, ce qui est peut-être une évolution liée à une demande sociale plus importante ${ }^{64}$. Des arithmétiques marchandes, le texte du manuscrit Paris, BnF, fr. 1339 est sans doute celui qui présente le plus de liens directs avec les cautelae. Il distingue clairement les problèmes où il peut appliquer la règle $\mathrm{d}$ ' « apposition remotion », d'une série qu'il intitule « questions de plaisance » (f. 69v) et dans laquelle il ne place que des devinettes ${ }^{65}$. L'écriture par problème de ce manuscrit est fortement marquée par une structure « question » et « réponse », mais aussi et sans doute par une mise en règle, c'est-à-dire une distinction des problèmes en fonction des méthodes utilisées pour les résoudre. Aussi, si l'idée de A. Heeffer, selon laquelle l'utilisation de l'algèbre laisse sous silence certains problèmes, est juste, elle n'est pas suffisante. Depuis le XIII' siècle au moins, on sait donner une solution à des problèmes que nous rangerions, aujourd'hui, sous la dénomination d'algébriques. On regroupe les problèmes linéaires du premier degré et on sait les associer à d'autres qui nécessitent les mêmes méthodes. Pourtant, ces problèmes continuent d'être présents dans les cautelae jusqu'à la fin du Moyen Âge. Ni les exemples que l'on connaît de reclassement, ni les règles utilisées pour les résoudre ne les font disparaître de ces recueils. Pour expliquer cette situation, il faut, sans doute, considérer

63. Cette règle est présente dans 8 ouvrages dont voici une liste chronologique : un traité anonyme, le Compendi del art de l'algorisme, contenu dans un manuscrit produit à Pamiers vers 1420-30 (Paris, BnF, fr. 4140), édité par J. Sesiano, L'Arithmétique de Pamiers : traité mathématique en langue d'oc du $\mathrm{Xv}^{\mathrm{e}}$ siècle. Lausanne, 2018 ; le traité contenu dans le ms. Paris, $\mathrm{BnF}$, fr. 1339, écrit vers 1460 ; le premier traité d'un ms. de Cesena, Bibl. Malatestiana, S-XXVI-6 (1476), intitulé Traicté de la praticque d'algorisme ; le Compendy de la praticque des nombres, deuxième traité de ce même ms. de Cesena, plus théorique et dont l'auteur est Barthélemy de Romans (1476), édité par M. SPIESSER, Une arithmétique commerciale $d u \mathrm{XV}^{\mathrm{e}}$ siècle : le Compendy de la practique des nombres de Barthélemy de Romans, Turnhout, 2003 ; le Triparty en la science des nombres de Nicolas Chuquet (Paris, BnF, fr. 1346), daté de 1484 ; le Kadran aux marchans de Jean Certain (Paris, Arsenal, 2904) daté de 1485 ; un ouvrage anonyme d'arithmeticque conservé aujourd'hui à la médiathèque de Nantes sous la cote 456 (1488) ; et un texte catalan, la Suma de la art de arismetica de Francesch Sanct Climent, imprimée à Barcelone (1482).

64. On peut prendre l'exemple des devinettes contenues dans le ms. de Chantilly, Musée Condé, 465, éditées par B. Roy, Devinettes françaises du Moyen Âge, Montréal/Paris, 1977. Ces adevinaux amoureux se présentent sous la forme questions-réponses et font en partie usage de ce qu'offrent les recueils mathématiques.

65. Une démarche un peu similaire peut-être observée dans le Traicté de la praticque d'algorisme du ms. Cesena, Biblioteca communale Malatestiana, S-XXVI-6 (1476), où l'on trouve une partie intitulée : "S'ensuivent les jeux et esbatemens d'algorisme » (f. 113r), qui se structure à la fois en désignant les problèmes sous la forme d'une expression figée et des opérations nécessaires à leur solution, par exemple : «S'ensuit le jeu des choses egales qui se fait par addition et sustraction » (f. 113r) ou « S'ensuit le jeu de l'anel » (f. 115r). 
qu'ils prennent place dans l'architecture du savoir mathématique universitaire dans la durée. Ces cautelae appartiennent à la culture mathématique de cette époque. L'observation fine, sur le temps long des réappropriations dont les problèmes de ces recueils ont fait l'objet permettrait, peut-être, d'appréhender l'évolution de la fonction de ces recueils dans les mathématiques médiévales. Ce qui suppose de reprendre le questionnement du statut des problèmes dans les textes universitaires en prêtant une attention nouvelle à leurs résonnances intertextuelles.

Avec le passage d'un enseignement au sein des écoles cathédrales à l'enseignement universitaire émerge un nouveau type de recueils ${ }^{66}$. Il est possible de délimiter le " genre » des cautelae avec ses propres caractéristiques. Le corpus que nous avons isolé n'est certes pas clos, mais il est le résultat d'une activité réelle et peut constituer un véritable terrain d'étude. On a cherché à analyser ce corpus dans une perspective plus large que celle de G. Beaujouan et sous un angle différent de celui de M. Folkerts, en testant sur eux l'hypothèse d'une mise en série et en tenant compte de la circulation des problèmes à l'intérieur du corpus d'étude. Cette hypothèse, qui s'appuie sur la typologie proposée par M. Folkerts, a permis d'observer la circulation d'autres traditions au sein de ces recueils. Celle des Propositiones ad acuendos iuvenes semble forte, mais elle n'est pas la seule. Différents héritages se mélangent dans ce type de recueils qui continuent en même temps à évoluer de façon indépendante. On a aussi souligné la difficulté de cette typologie pour donner sens aux successions de problèmes contenus dans les recueils. Il semble qu'il y ait des séquences identifiables au début de ces textes, mais que par la suite la diversité des permutations rend la comparaison difficile. On peut y voir une adaptation de ces recueils à des démarches particulières, comme l'atteste le manuscrit Paris, BnF, lat. 14070, ou comme un stock où l'on viendrait puiser ce que l'on cherche. Il faudrait poursuivre le dépouillement afin de vérifier ce constat d'une identité plus forte au XIV $v^{\mathrm{e}}$ siècle des séquences organisées. Nous voilà bien loin de l'idée préconstruite d'une structure qui aurait été fixée très tôt. Cet ensemble documentaire donne le sentiment, au contraire, d'une structure en mouvement ${ }^{67}$. L'analyse de la circulation révèle des liens denses entre

66. G. Beaujouan, «L'enseignement de l'arithmétique élémentaire à l'Université de Paris... »; M. FOLKERTS, « Mathematische Aufgabensammlungen aus dem ausgehenden Mittelalter... » La croissance du nombre des exemplaires pourrait trouver une de ses causes dans ce lien avec le développement universitaire.

67. F. ACERTO, « "Aguzzar l'ingegno dei Giovanni”. Jeux, mathématiques et violences symboliques au Quattrocento », Ludica, 21-22 (2016), p. 135-141. L'auteure propose de s'intéresser aux modes de transmissions qui lui permettraient d'envisager l'émergence de « formes spécifiques de rationalité scientifique », mais aussi la façon dont on peut mettre en jeu. 
ces recueils. Il est possible de les interpréter en termes de diffusion, mais on peut aussi remarquer un de ses effets, soit la construction d'une forme de « culture commune ${ }^{68} »$. Plusieurs éléments concourent à cette observation, comme la formulation des problèmes eux-mêmes. Il arrive qu'ils soient présentés sous une forme de locution ou de phrasème. Ils sont mobilisés hors de ces cautelae et cela dès le XIII ${ }^{\mathrm{e}}$ siècle. On a pu l'observer avec le De regulis generalibus. Une étude précise des relations avec le Liber Abaci (1202) de Léonard de Pise permettrait de mieux éclairer ces échanges ${ }^{69}$. En effet, cet ouvrage contient ces problèmes, non pas forcément sous une forme de série monolithique, mais en les mobilisant dans différentes parties de son livre, dans une structure organisée autour de règles de résolution.

Par ailleurs, malgré les difficultés que nous avons pour les dater de manière précise, les problèmes contenus dans ces cautelae n'ont jamais cessé de circuler, d'être réappropriés et remis en contexte. On connaît jusqu'au $\mathrm{XV}^{\mathrm{e}}$ siècle des ouvrages d'arithmétiques marchandes jugeant nécessaire de faire une place à ce type de problèmes, mais aussi à l'idée de récréation qu'incarnent ces recueils. Peut-on qualifier, avec G. Beaujouan, l'évolution de ces problèmes au $\mathrm{XV}^{\mathrm{e}}$ siècle de « courtoise »? Ne faut-il pas voir dans cette évolution la convergence d'une culture de bourgeois, qui intègre celle venant d'un monde considéré comme plus savant? Il me semble que, dans un certain nombre de cas comme celui des adevinaux amoureux étudiés par Bruno Roy, c'est bien l'énoncé qui véhicule la culture. Le choix du mode de résolution est adapté à la finalité du texte, qui est ici le plaisir du public. Quoiqu'il en soit, cet ensemble de problèmes a profondément marqué l'apprentissage des mathématiques occidentales sur le long terme.

On connaît peu de choses sur l'utilisation de ces recueils dans le cadre d'un enseignement pour le Moyen Âge, mais il me semble acquis qu'ils furent utilisés. Étaient-ils utilisés comme des curiosités, pour éveiller par exemple les bacheliers ès arts, des élèves âgés entre 14 et 20 ans ? Ou par des élèves d'écoles dans lesquelles les universitaires pouvaient enseigner? Ces séries de problèmes mobilisent une activité mathématique centrée sur la manipulation des nombres, le raisonnement, la compréhension d'un énoncé, la possibilité de solutions multiples. Ils permettent de s'initier à l'idée de combinaisons, et la versification joue à ce propos un rôle important. Il semble acquis qu'un certain nombre de textes ont cherché à associer le

68. Nous avons pu déjà aborder cette idée dans S. LAMAssé, « Relationships Between French Practical Arithmetics and Teaching », dans A. Bernard et C. Proust éd., Ancient Sources and Teaching Context : Problems and Perspectives, Dordrecht, 2014.

69. Nous n'avons pas envisagé dans cet article les relations possibles entre ces recueils et des textes où certains de ces problèmes apparaissent comme le Liber augmentis et diminutionis, le Liber Abaci de Léonard de Pise ou le Liber Mahameleth. C'est un sujet vers lequel nous souhaitons à présent nous tourner, soit celui des premiers développements de ce corpus. 
contenu des problèmes avec celui de l'Algorismus de Sacrobosco. Il faut sans doute aller plus loin en continuant le dépouillement et prêter une attention plus grande à une analyse codicologique précise qui livrerait des informations précieuses sur le contexte de ces cautelae.

\section{Stéphane Lamassé - Université Paris Sorbonne - LAMOP, UMR 8589}

Les cautelae : un corpus de problèmes mathématiques, entre collection, série et culture mathématique

Cet article se propose de reconsidérer un ensemble de textes regroupant

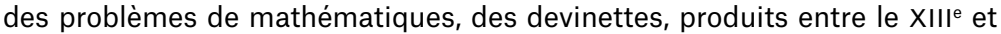
la fin du XVe siècle dont une des origines sont les propositiones ad acuendos juvenes. En adoptant un regard critique sur la production historiographique dont ils ont fait l'objet, on isole un ensemble de recueils en mettant en avant leurs éléments de cohérence. De cet ensemble on peut faire un corpus, lequel replacé dans une perspective diachronique peut nous permettre de faire des hypothèses sur les usages dont ces recueils ont pu faire l'objet.

Alcuin, algorisme, enseignement médiéval, jeux mathématiques, mathématiques

\section{Cautelae: A Corpus of Mathematical Problems, between Collection, Series and Mathematical Culture}

This article analyzes a number of texts that group mathematical problems and riddles. They were produced between the thirteenth and the late fifteenth centuries, and one of their origins is Propositiones ad Acuendos Juvenes (Problems to Sharpen the Young). By adopting a critical perspective on the historiographical production of which they have been the subject, it is possible to isolate a group of collections by highlighting their elements of coherence. From this group, we can create a corpus, which, placed in a diachronic perspective, allows us to make hypotheses on the uses of which these collections might have been the object.

Alcuin, algorism, mathematical games, mathematics, medieval teaching 